\title{
SARS-CoV-2 protein subunit vaccination elicits potent neutralizing antibody responses
}

\author{
Marco Mandolesi ${ }^{1, *}$, Daniel J. Sheward ${ }^{1,2, *,}$, Leo Hanke ${ }^{1}$, Junjie Ma ${ }^{1}$, Pradeepa Pushparaj $^{1}$, Laura Perez Vidakovics ${ }^{1}$, \\ Changil Kim ${ }^{1}$, Karin Loré ${ }^{3}$, Xaquin Castro Dopico ${ }^{1}$, Jonathan M. Coquet ${ }^{1}$, Gerald McInerney ${ }^{1}$, Gunilla B. Karlsson \\ Hedestam $^{1, \dagger, \bowtie}$, and Ben Murrell ${ }^{1, \dagger, \bigotimes}$ \\ ${ }^{1}$ Department of Microbiology, Tumor and Cell Biology, Karolinska Institutet, Stockholm, Sweden \\ ${ }^{2}$ Division of Medical Virology, Institute of Infectious Diseases and Molecular Medicine, University of Cape Town, South Africa \\ ${ }^{3}$ Department of Medicine, Solna, Karolinska Institutet, Stockholm, Sweden \\ ${ }^{*}$ These authors contributed equally \\ † These authors contributed equally
}

\begin{abstract}
The outbreak and spread of SARS-CoV-2 (Severe Acute Respiratory Syndrome coronavirus 2), the cause of coronavirus disease 2019 (COVID-19), is a current global health emergency and a prophylactic vaccine is needed urgently. The spike glycoprotein of SARS-CoV-2 mediates entry into host cells, and thus is a target for neutralizing antibodies and vaccine design. Here we show that adjuvanted protein immunization with SARS-CoV-2 spike trimers, stabilized in prefusion conformation ${ }^{1}$, results in potent antibody responses in mice and rhesus macaques with neutralizing antibody titers orders of magnitude greater than those typically measured in serum from SARS-CoV-2 seropositive humans. Neutralizing antibody responses were observed after a single dose, with exceptionally high titers achieved after boosting. Furthermore, neutralizing antibody titers elicited by a dose-sparing regimen in mice were similar to those obtained from a high dose regimen. Taken together, these data strongly support the development of adjuvanted SARS-CoV-2 prefusionstabilized spike protein subunit vaccines.
\end{abstract}

Correspondence: daniel.sheward@ki.se, gunilla.karlsson.hedestam@ki.se, benjamin.murrell@ki.se

\section{Introduction}

As of 22nd July 2020, at least 15 million cases of SARSCoV-2 infection have been confirmed, with over 615,000 COVID-19 related deaths recorded ${ }^{2}$. Cases continue to spread globally despite unprecedented public health measures and lockdowns. An effective prophylactic vaccine is urgently required. Adjuvanted recombinant protein subunit vaccines have excellent safety profiles and represent a proven vaccine platform for eliciting protective immune responses to viral infections, including human papillomavirus (HPV), hepatitis B virus (HBV), and influenza A virus (IAV).

The spike glycoprotein of SARS-CoV-2 mediates receptor binding and entry into target cells, and is the primary target for vaccine design. The receptor binding domain (RBD) is a stable subunit within the spike glycoprotein (Fig. 1A) responsible for ACE2 binding ${ }^{3-7}$, that can be expressed as an independent domain ${ }^{7-9}$. While the RBD is a major target for neutralizing antibodies ${ }^{10-12}$, antibodies specific for spike epitopes outside of the RBD are also capable of neutralization $^{13,14}$.

\section{Results}

To evaluate the use and immunogenicity of recombinant protein subunit vaccines for SARS-CoV-2 we immunized C57BL/6J mice $(\mathrm{N}=24)$ with either the spike ectodomain or RBD, expressed in 293-F cells. The RBD domain was expressed as an Fc-fusion protein, which was cleaved and the RBD subsequently purified by size-exclusion chromatography. The spike ectodomain was expressed as a prefusionstabilized variant with a $\mathrm{C}$-terminal $\mathrm{T} 4$ trimerization domain, and the introduction of two stabilizing Proline mutations in the C-terminal S2 fusion machinery ${ }^{1,15,16}$. Highly pure and homogenous spike glycoprotein trimers were obtained by affinity purification followed by size-exclusion chromatography (Extended Data Fig. 1). Cryo-EM analysis of the spike preparation demonstrated that it was well-folded and maintained in the trimeric, prefusion conformation ${ }^{17}$, consistent with the original report ${ }^{1}$.

Mice were immunized with varying doses of antigen (range: $5 \mu \mathrm{g}-50 \mu \mathrm{g}$ ) in Addavax (InvivoGen), a squalene-based oilin-water emulsion analogous to MF59. MF59 is licensed, safe and effective in humans ${ }^{19}$, and increases the immunogenicity of an influenza vaccine in the elderly ${ }^{20}$. Mice were boosted twice, at 3-week intervals, beginning 4 weeks after prime (Fig. 1A). In both the low-dose $(5 \mu \mathrm{g})$ and highdose $(25 \mu \mathrm{g})$ groups, a single immunization with prefusionstabilized spike elicited a strong spike-specific IgG antibody response, detected by ELISA (Extended Data Fig. 2), as early as 4 weeks following the first immunization. RBD was less immunogenic, with weak to no detectable responses after the initial prime. However, seroconversion was evident in all mice after the first boost with RBD.

To determine whether the antibody responses elicited were neutralizing, we used a SARS-CoV-2 pseudotyped virus neutralization assay. In spike-immunized mice, neutralizing responses (ID50 100) were already detectable in four of ten mice after a single dose (Fig. 1B and Extended Data Fig. 3). All spike-immunized mice developed potent neutralizing antibody responses (median ID50 1,600) after the first boost, which further increased in potency (median ID50 25,000) after the second boost. In contrast, RBD-immunized mice developed consistent neutralizing antibody responses only after the second boost, with a median ID50 neutralizing anti- 

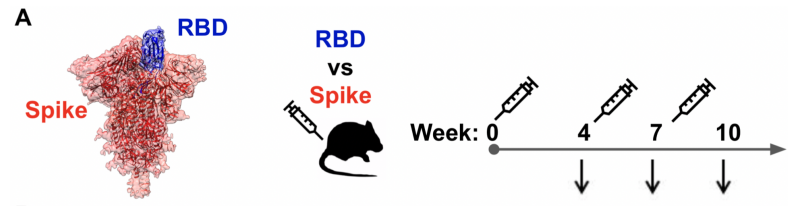

B

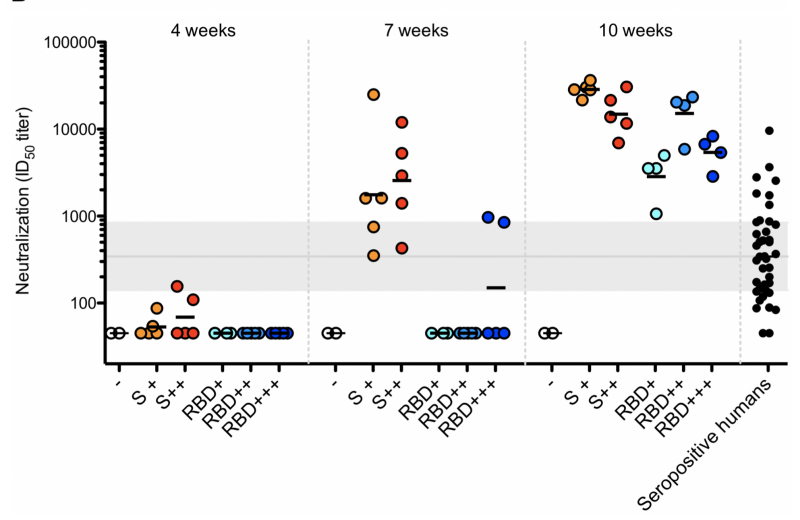

Fig. 1. Protein subunit vaccines elicit potent neutralizing antibodies in mice. A. Two SARS-CoV-2 protein immunogens were evaluated: stabilized spike trimer, and receptor binding domain (RBD). Mice $(\mathrm{N}=24)$ were immunized and humoral immune responses were followed longitudinally to compare stabilized spike vs RBD immunogens at several doses. B. Serial dilutions of serum from immunized mice were assessed for neutralization of SARS-CoV-2 pseudotyped lentiviruses harbouring a luciferase reporter gene, and the ID50 titers were calculated as the reciprocal dilution where infection (RLU) was reduced by $50 \%$ relative to infection in the absence of serum. The geometric mean ID50 for each group is displayed. - (unimmunized mice - open circles); $\mathrm{S}_{+}(5 \mu \mathrm{g}$ stabilized spike - orange); $\mathrm{S}++(25 \mu \mathrm{g}$ stabilized

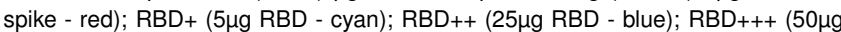
RBD - navy). ID50 titers below the limit of detection (45 or 90 depending on sample availability) are displayed as 45. ID50 titers in seropositive donors (black) in Castro Dopico et al. ${ }^{18}$ determined using the same assay, and the median and interquartile range is highlighted in grey across the background.

body titer across all groups of 5,300. The substantial enhancement of the virus neutralizing activity observed after the second RBD boost warrants further investigation. Across all groups, spike-specific IgG titers correlated strongly with pseudovirus neutralization, though neutralization was detectable only above a threshold EC50 (Extended Data Fig $5 \mathrm{~A})$. Interestingly, RBD medium $(25 \mu \mathrm{g})$ and high $(50 \mu \mathrm{g})$ doses elicited neutralizing antibody responses with similar ID50 values to those from the spike groups, but with a significantly flatter slope $(\mathrm{p}=0.0002)$, such that they were less neutralizing at higher concentrations (see Extended Data Fig. 4). This could potentially be a result of epitopes on soluble RBD being subject to competition in the context of intact spike trimer. This also suggests an additional advantage of spike immunization over RBD alone, as incomplete neutralization may compromise sterilizing immunity.

Next, we immunized three rhesus macaques (Macaca mulatta) with adjuvanted trimeric prefusion-stabilized spike glycoprotein over approximately 4-week intervals (Fig. 2A), and characterized the titers and kinetics of binding and neutralizing antibodies. Macaques were immunized intramuscularly with $100 \mu \mathrm{g}$ of spike protein in $75 \mu \mathrm{g}$ Matrix-M, a saponin-based adjuvant developed for clinical use ${ }^{21}$ (Novavax $A B$ ). Neutralizing antibody responses were already detectable 2 weeks after a single dose (ID50 titers ranging from 150-420) with titers retained at 4 weeks (Fig. 2C). Two weeks after a first boost, the neutralizing antibody responses were extremely potent, exceeding ID50 titers of 20,000 in all three macaques. An additional boost 3 weeks later did not raise the neutralization potency above that obtained with just two immunizations, suggesting a third dose is not needed to achieve highly potent neutralizing antibody responses following spike protein-based vaccination.

After two prefusion-stabilized spike protein immunizations, geometric mean neutralizing antibody titers in macaques were approximately two orders of magnitude more potent than that of sera from SARS-CoV-2 seropositive humans (Fig. 2C). These were also substantially higher than those elicited by other immunization platforms that afforded macaques partial or complete protection from challenge ${ }^{22,23}$. In three recent phase I human trials, neutralizing antibody titers elicited by mRNA or adenovirus-vectored vaccines were similar to ${ }^{24,25}$ or well below ${ }^{26}$ those from convalescent sera.

The spike-directed $\mathrm{IgG}$ binding titers elicited in the macaques (Fig. 2B) correlated strongly with the virus neutralizing activity (R2 $=0.9478$, Extended Data Fig. 5) and the rapid development of potent virus neutralizing antibody responses in macaques after a single dose suggested a minimal requirement for somatic hypermutation (SHM) of the response. This is consistent with recent data showing that neutralizing monoclonal antibodies isolated from SARS CoV-2 convalescent individuals display low levels of SHM ${ }^{12,27,28}$. The polyclonality, antibody germline VDJ usage, and level of SHM that characterizes vaccine-induced SARS CoV-2 spike-induced antibody responses will be a matter of interest, both in the macaque model and in human vaccine trials.

\section{Discussion}

Neutralizing antibody titers have varied substantially across different vaccine platforms. In animal models, inactivated virus, DNA, and adenovirus-vectored vaccines elicited neutralizing antibody titers similar to or lower than those seen in convalescent sera $22,23,29$. While high dose mRNA-based immunizations elicited potent neutralizing antibody responses in mice ${ }^{30}$, neutralizing antibody titers elicited in phase I human trials were markedly lower ${ }^{25}$. Immunization of mice with mRNA encoding stabilized spike led to reduced or undetectable viral loads in the lungs and nasal turbinates, in a dose-dependent manner, and susceptible transgenic mice were protected from lethal challenge ${ }^{30}$. Other preclinical vaccine studies using genetic platforms elicited immune responses that protect against disease but not against infection. For example, RBD- and spike-encoding DNA vaccines led to reduced viral loads in the nose and lungs of challenged macaques $^{22,23}$.

Titers of neutralizing antibodies correlate strongly with protection in a number of vaccine settings ${ }^{31-33}$. For SARS-CoV2 , passive transfer of neutralizing monoclonal antibodies provided partial or complete protection of animal models, in a dose-dependent manner ${ }^{11,34,35}$. Here, we show in mice and macaques that neutralizing antibody titers elicited by both spike and RBD protein subunit immunization were orders of magnitude higher than those we detected in SARS-CoV- 
A

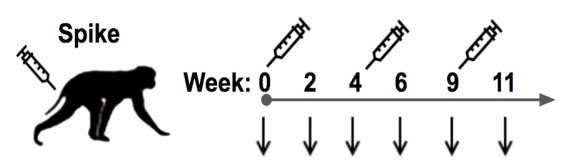

C

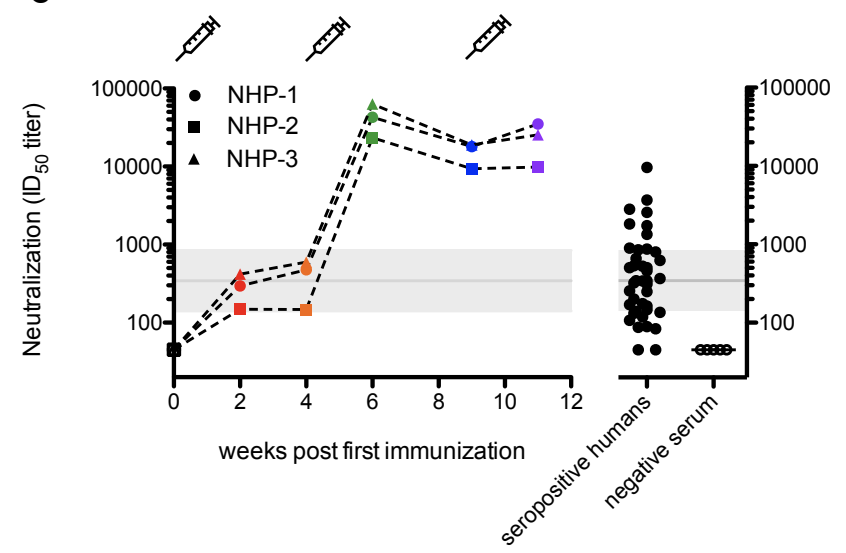

B

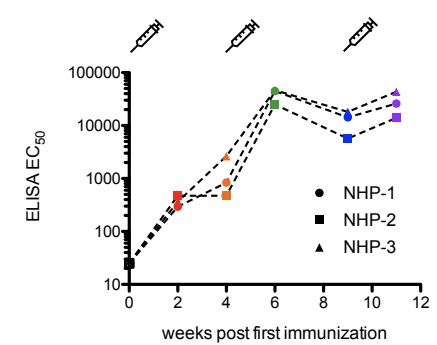

D

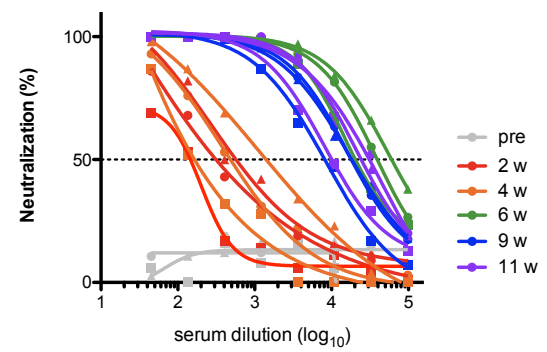

Fig. 2. Prefusion-stabilized SARS-CoV-2 spike protein subunit vaccine reproducibly elicits potent neutralizing antibody responses in non-human primates. A. Macaque $(\mathrm{N}=3)$ immunization and sampling schedule. Syringes indicate the timing of immunizations, and arrows denote times at which blood was drawn. B. Longitudinal spike directed IgG responses. C. Longitudinal neutralization potency over the course of the study. Neutralization below the assay limit of detection (45) is plotted as 45. Shaded band corresponds to the median and interquartile range of the neutralization potency observed in seropositive human donors using the same assay ${ }^{18}$, shown on the right. D. Neutralization curves depicting percent neutralization against serum dilution.

2 seropositive humans. This reinforces that vaccination can induce more reliable immunity in the population than natural infection. Since it is expected that binding and neutralizing antibody titers decrease from their peak with time, high initial titers will most likely translate into longer lasting protection. At least 19 candidate SARS-CoV-2 vaccines have rapidly entered clinical trials ${ }^{36}$. These include DNA and RNAbased platforms (Moderna ${ }^{25}$, Inovio, BioNTech/Pfizer ${ }^{37}$ ), adenovirus vectored vaccines (CanSino ${ }^{26}$, University of Oxford/AstraZeneca ${ }^{24}$, Johnson \& Johnson), inactivated virus vaccines (Sinovac, Wuhan Institute of Biological Products, Beijing Institute of Biological Products), and recombinant protein vaccines (Novavax ${ }^{38}$, Anhui Zhifei Longcom Biopharmaceutical, Clover Biopharmaceuticals/GlaxoSmithKline). Given the urgency in the current pandemic, vaccines will require rapid mobilization on a global scale. As a result, safety is an important consideration, and the use of proven vaccine platforms (particularly in the elderly) would be advantageous. DNA and RNA vaccines are amenable to large-scale and rapid vaccine manufacture; however, no nucleic acid or recombinant adenovirus vaccines are approved for human use, and therefore remain largely untested at scale.

Recombinant protein subunit vaccines have been developed successfully for Influenza, HPV, and HBV, and are also being explored for SARS-CoV-2 vaccination, including three candidate vaccines that have already entered phase I clinical trials. Here we show in animal models (mice and macaques) that immunization with RBD or prefusion-stabilized trimeric SARS-CoV-2 spike proteins elicits potent neutralizing antibody responses. Further, we find that neutralizing responses are more readily elicited and more potent with the stabilized trimeric spike glycoprotein than with RBD. Importantly, we show that neutralizing antibodies were elicited across animal models, using different immunization routes and with different adjuvants, highlighting that the SARS-CoV-2 spike protein represents a robust immunogen. Importantly, significant neutralizing antibody titers arose in non-human primates after a single dose with an adjuvanted spike subunit protein vaccine.

Production of prefusion-stabilized spike at an industrial scale to meet global demand in a rapid time frame may be challenging. However, a number of groups have recently identified additional stabilizing mutations and protocols that dramatically improve protein stability and expression yields ${ }^{39-41}$. Further, we show in mice that a dose-sparing regimen with 5 -fold less spike antigen elicited neutralizing antibodies at a similar titer. The potential to leverage existing infrastructure and manufacturing capacity for licensed vaccines could also accelerate production and rollout.

Here we report that mice and macaques immunized with adjuvanted prefusion-stabilized spike developed potent neutralizing antibody responses. These are stronger than those previously reported to confer partial or complete protection in animal models, and substantially more potent than those from three recent phase I human trials of mRNA or adenovirusvectored vaccines ${ }^{24-26}$. Taken together, these results support the development of protein subunit vaccines to immunize against SARS-CoV-2.

\section{ACKNOWLEDGEMENTS}

We thank Dr. Bengt Eriksson and all personnel at Astrid Fagraeus laboratory for expert assistance with rhesus macaques. We also thank Novavax, AB, Uppsala, 
bioRxiv preprint doi: https://doi.org/10.1101/2020.07.31.228486; this version posted July 31, 2020. The copyright holder for this preprint (which was not certified by peer review) is the author/funder, who has granted bioRxiv a license to display the preprint in perpetuity. It is made available under aCC-BY 4.0 International license.

Sweden, for generously making the Matrix-M adjuvant available. We additionally thank James Voss and Deli Huang for reagents. This project has received funding from the European Union's Horizon 2020 research and innovation programme under grant agreement No. 101003653 (CoroNAb), to GBKH, GM, and BM, and from the Swedish Research Council to GBKH, GM, and BM.

\section{AUTHOR CONTRIBUTIONS}

Conceptualization, DJS, MM, GBKH, BM; Formal Analysis, DJS, MM, GBKH, BM; Investigation, DJS performed the neutralization assays and the mouse ELISAs. JM performed the mouse immunizations and bleeds. MM, PP coordinated the NHP immunizations, processed samples and performed ELISAs; Resources, LH and LPV produced RBD and spike immunogens, CK and DJS produced pseudovirus, XCD contributed data from seropositive humans; Visualization, DJS, LH, BM; Writing Original Draft, DJS, BM; Writing - Review \& Editing, DJS, KL, JC, GM, BM, GBKH; Funding Acquisition, BM, GBKH, GM; Supervision, BM, GBKH, JC, GM.

\section{METHODS}

Ethics statement: The animal work was conducted with the approval of the regional Ethical Committee on Animal Experiments (Stockholms Norra Djurförsöksetiska Nämnd). All animal procedures were performed according to approved guidelines.

Protein production: The plasmid for expression of the SARS-CoV-2 prefusionstabilized spike ectodomain ${ }^{1}$ was kindly provided by Jason McLellan. This plasmid was used to transiently transfect freestyle 293-F cells using the FreeStyle MAX reagent (Thermo Fisher Scientific). The spike ectodomain was purified from filtered supernatant on Streptactin XT resin (IBA Lifesciences), followed by size-exclusion chromatography on a Superdex 200 in $5 \mathrm{mM}$ Tris $\mathrm{pH} 8,200 \mathrm{mM} \mathrm{NaCl}$.

The RBD domain (RVQ-VNF) of SARS-CoV-2 was cloned upstream of an enterokinase cleavage site and a human Fc. This plasmid was used to transiently transfect FreeStyle 293F cells using the FreeStyle MAX reagent. The RBD-Fc fusion was purified from filtered supernatant on Protein G Sepharose (GE Healthcare) and cleaved using bovine enterokinase (GenScript) leaving a FLAG-tag on the Cterminus of the RBD. Enzyme and Fc-portion was removed on His-Pur Ni-NTA resin and Protein G Sepharose respectively, and the RBD was purified by size-exclusion chromatography on a Superdex 200 in $5 \mathrm{mM}$ Tris pH 8, $200 \mathrm{mM} \mathrm{NaCl}$. Proteins were re-buffered into PBS prior to immunization.

Mice: C57BL/6J (Jackson Laboratory) wild-type mice were housed and bred at Karolinska Institutet animal facility. Immunogens were diluted in sterile PBS, emulsified in AddaVax (InvivoGen) and injected subcutaneously (s.c.) in the flanks of mice. Each arm contained 5 mice, except the low-dose RBD group which had 4. Two control mice were not immunized. Tail bleeds were taken prior to each immunization. Whole blood was allowed to clot at room temperature, and serum was separated by centrifugation, heat inactivated at $56^{\circ} \mathrm{C}$ for 1 hour, and stored at $-20^{\circ} \mathrm{C}$ until use.

Macaques: Two female and one male rhesus macaques (Macaca mulatta) of Chinese origin, 4-5 years old, were housed at the Astrid Fagraeus Laboratory at Karolinska Institutet. Housing and care procedures complied with the provisions and general guidelines of the Swedish Board of Agriculture. The facility has been assigned an Animal Welfare Assurance number by the Office of Laboratory Animal Welfare (OLAW) at the National Institutes of Health (NIH). The macaques were housed in groups in $14 \mathrm{~m} 3$ enriched cages. They were habituated to the housing conditions for more than six weeks before the start of the experiment and subjected to positive reinforcement training in order to reduce the stress associated with experimental procedures. All immunizations and blood samplings were performed under sedation with 10-15 mg/kg ketamine (Ketaminol $100 \mathrm{mg} / \mathrm{ml}$, Intervet, Sweden) administered intramuscularly (i.m.). The macaques were weighed at each sampling. All animals were confirmed negative for simian immunodeficiency virus (SIV), simian T cell lymphotropic virus, simian retrovirus type D and simian herpes $\mathrm{B}$ virus.

For macaque immunizations, stabilized spike trimer $(100 \mu \mathrm{g})$ was mixed in $75 \mu \mathrm{g}$ of Matrix-M (Novavax AB). Macaques were immunized intramuscularly (i.m.) with half of the dose administered in each quadricep.

Mouse ELISAs: ELISA plates (Nunc MaxiSorp, Thermo Fisher Scientific) were coated overnight at $4^{\circ} \mathrm{C}$ with $100 \mu$ of prefusion-stabilized spike protein at a concentration of $1 \mu \mathrm{g} / \mathrm{ml}$ in $1 \times$ PBS. Plates were blocked for 90 minutes at room temperature with $200 \mu \mathrm{l}$ of a blocking solution containing $2 \%(\mathrm{w} / \mathrm{v})$ non-fat milk powder in 1X PBS, and washed 6 times with 1X PBS supplemented with $0.05 \%$ Tween 20 (PBS-T). Serum samples serially diluted in blocking solution were added and plates were incubated overnight at $4^{\circ} \mathrm{C}$. Plates were washed 6 times with PBS-T, and $100 \mu \mathrm{l}$ of a goat anti-Mouse lgG horseradish peroxidase (HRP) conjugated secondary antibody (Southern Biotech) diluted 1:5,000 in PBS-T was added to each well. Plates were washed 6 times with PBS-T, developed for 15 minutes at room temperature using $200 \mu \mathrm{l}$ per well of peroxidase substrate (o-phenylenediamine dihydrochloride, SIGMAFAST, SigmaAldrich), and read at $450 \mathrm{~nm}$ in an Asys Expert 96 plate reader (Biochrom). EC50 titers were calculated using a Bayesian logistic curve fitting approach, allowing plate-specific minimum and maximum sigmoid parameters to account for differences between plates, and sample specific slope and offset parameters. EC50 titers were calculated from the posterior median value midway between the plate minimum and maximum.

Macaque ELISAs: ELISA plates were coated with prefusion-stabilized spike protein as above, washed, and blocked with $5 \%(\mathrm{w} / \mathrm{v})$ non-fat milk powder in $1 \mathrm{x}$ PBS. Thereafter, 10-fold serial dilutions (starting at 1:200) of plasma in blocking buffer were added, and plates were incubated for 2 hours at room temperature. Plates were washed and antibody-antigen interaction was detected using HRP-conjugated anti-monkey IgG Fc (\#GAMon/lgG(Fc)/PO Nordic MUbio) diluted 1:20,000 in PBST. Plates were developed using $50 \mu \mathrm{l}$ of 3,3',5,5"-tetramethylbenzidine substrate so- lution (Invitrogen) per well and stopped using $50 \mu \mathrm{l}$ of $1 \mathrm{M}$ sulphuric acid per well. OD was read at 450nm in an Asys Expert 96 plate reader (Biochrom). Every washing step was performed with $0.05 \%$ PBS-T. All experiments were conducted in triplicates. EC50 titers were calculated as for the mice.

Pseudotyped neutralization assays: Pseudotyped neutralization assays were adapted from protocols previously validated to characterize the neutralization of HIV $^{42}$ but with the use of HEK293T-ACE2 cells, as previously described ${ }^{17}$. All cell lines were cultured in a humidified $37^{\circ} \mathrm{C}$ incubator $(5 \% \mathrm{CO})$ in Dulbecco's Modified Eagle Medium (Gibco) supplemented with 10\% Fetal Bovine Serum and $1 \%$ Penicillin/Streptomycin, and were passaged when nearing confluency using $1 \mathrm{X}$ Trypsin-EDTA. All cell lines tested negative for mycoplasma by PCR. Pseudotyped lentiviruses displaying the SARS-CoV-2 spike protein (harboring an 18 amino acid truncation of the cytoplasmic tail) and packaging a luciferase reporter gene were generated by the co-transfection of HEK293T cells using Lipofectamine 3000 (Invitrogen) per the manufacturer's protocols. Media was changed 12-16 hours after transfection, and pseudotyped viruses were harvested at 48- and 72-hours post transfection, filtered through a $0.45 \mu \mathrm{m}$ filter, and stored at $-80^{\circ} \mathrm{C}$ until use. Pseudotyped viruses sufficient to generate 100,000 RLUs were incubated with serial dilutions of serum for $60 \mathrm{~min}$ at $37^{\circ} \mathrm{C}$ in a 96-well plate, and then 15,000 HEK293TACE2 cells were added to each well. Plates were incubated at $37^{\circ} \mathrm{C}$ for 48 hours, and luminescence was then measured using Bright-Glo (Promega) per the manufacturer's protocol, on a GM-2000 luminometer (Promega). ID50 titers were calculated as the reciprocal serum dilution at which RLUs were reduced by $50 \%$ relative to control wells in the absence of serum.

\section{Bibliography}

1. Daniel Wrapp, Nianshuang Wang, Kizzmekia S Corbett, Jory A Goldsmith, Ching-Lin Hsieh, Olubukola Abiona, Barney S Graham, and Jason S McLellan. Cryo-EM structure of the 2019-nCoV spike in the prefusion conformation. bioRxiv, February 2020.

2. COVID-19 situation update worldwide, as of 3 july 2020. https : //www . ecdc . europa . eu/en/geographical-distribution-2019-ncov-cases, . Accessed: 2020-73.

3. Markus Hoffmann, Hannah Kleine-Weber, Simon Schroeder, Nadine Krüger, Tanja Herrler, Sandra Erichsen, Tobias S Schiergens, Georg Herrler, Nai-Huei Wu, Andreas Nitsche, Marcel A Müller, Christian Drosten, and Stefan Pöhlmann. SARS-CoV-2 cell entry depends on ACE2 and TMPRSS2 and is blocked by a clinically proven protease inhibitor. Cell, 181(2): 271-280.e8, April 2020

4. Alexandra C Walls, Young-Jun Park, M Alejandra Tortorici, Abigail Wall, Andrew T McGuire, and David Veesler. Structure, function, and antigenicity of the SARS-CoV-2 spike glycoprotein. Cell, 181(2):281-292.e6, April 2020.

5. Michael Letko, Andrea Marzi, and Vincent Munster. Functional assessment of cell entry and receptor usage for SARS-CoV-2 and other lineage B betacoronaviruses. Nat Microbiol, 5 (4):562-569, April 2020

6. Peng Zhou, Xing-Lou Yang, Xian-Guang Wang, Ben Hu, Lei Zhang, Wei Zhang, Hao-Rui Si, Yan Zhu, Bei Li, Chao-Lin Huang, Hui-Dong Chen, Jing Chen, Yun Luo, Hua Guo, RenDi Jiang, Mei-Qin Liu, Ying Chen, Xu-Rui Shen, Xi Wang, Xiao-Shuang Zheng, Kai Zhao, Quan-Jiao Chen, Fei Deng, Lin-Lin Liu, Bing Yan, Fa-Xian Zhan, Yan-Yi Wang, Geng-Fu Xiao, and Zheng-Li Shi. A pneumonia outbreak associated with a new coronavirus of probable bat origin. Nature, February 2020.

7. Jun Lan, Jiwan Ge, Jinfang Yu, Sisi Shan, Huan Zhou, Shilong Fan, Qi Zhang, Xuanling Shi, Qisheng Wang, Linqi Zhang, and Xinquan Wang. Structure of the SARS-CoV-2 spike receptor-binding domain bound to the ACE2 receptor. Nature, 581(7807):215-220, May 2020.

8. Swee Kee Wong, Wenhui Li, Michael J Moore, Hyeryun Choe, and Michael Farzan. A 193-amino acid fragment of the SARS coronavirus S protein efficiently binds angiotensinconverting enzyme 2. J. Biol. Chem., 279(5):3197-3201, January 2004.

9. Brian D Quinlan, Huihui Mou, Lizhou Zhang, Yan Guo, Wenhui He, Amrita Ojha, Mark S Parcells, Guangxiang Luo, Wenhui Li, Guocai Zhong, Hyeryun Choe, and Michael Farzan. The SARS-CoV-2 receptor-binding domain elicits a potent neutralizing response without antibody-dependent enhancement. April 2020.

10. Anna Z Wec, Daniel Wrapp, Andrew S Herbert, Daniel Maurer, Denise Haslwanter, Mrunal Sakharkar, Rohit K Jangra, M Eugenia Dieterle, Asparouh Lilov, Deli Huang, Longping V Tse, Nicole V Johnson, Ching-Lin Hsieh, Nianshuang Wang, Juergen H Nett, Elizabeth Champney, Irina Burnina, Michael Brown, Shu Lin, Melanie Sinclair, Carl Johnson, Sarat Pudi, Robert Bortz, 3rd, Ariel S Wirchnianski, Ethan Laudermilch, Catalina Florez, J Maximilian Fels, Cecilia M O'Brien, Barney S Graham, David Nemazee, Dennis R Burton, Ralph S Baric, James E Voss, Kartik Chandran, John M Dye, Jason S McLellan, and Laura M Walker. Broad sarbecovirus neutralizing antibodies define a key site of vulnerability on the SARSCoV-2 spike protein. bioRxiv, May 2020.

11. Thomas F Rogers, Fangzhu Zhao, Deli Huang, Nathan Beutler, Alison Burns, Wan-Ting $\mathrm{He}$, Oliver Limbo, Chloe Smith, Ge Song, Jordan Woehl, Linlin Yang, Robert K Abbott, Sean Callaghan, Elijah Garcia, Jonathan Hurtado, Mara Parren, Linghang Peng, Sydney Ramirez, James Ricketts, Michael J Ricciardi, Stephen A Rawlings, Nicholas C Wu, Meng Yuan, Davey M Smith, David Nemazee, John R Teijaro, James E Voss, Ian A Wilson, Raiees Andrabi, Bryan Briney, Elise Landais, Devin Sok, Joseph G Jardine, and Dennis R Burton. Isolation of potent SARS-CoV-2 neutralizing antibodies and protection from disease in a small animal model. Science, June 2020.

12. Davide F Robbiani, Christian Gaebler, Frauke Muecksch, Julio C C Lorenzi, Zijun Wang, Alice Cho, Marianna Agudelo, Christopher O Barnes, Anna Gazumyan, Shlomo Finkin, Thomas Hägglöf, Thiago Y Oliveira, Charlotte Viant, Arlene Hurley, Hans-Heinrich Hoffmann, Katrina G Millard, Rhonda G Kost, Melissa Cipolla, Kristie Gordon, Filippo Bianchini, Spencer T Chen, Victor Ramos, Roshni Patel, Juan Dizon, Irina Shimeliovich, Pilar Mendoza, Harald Hartweger, Lilian Nogueira, Maggi Pack, Jill Horowitz, Fabian Schmidt, Yiska 
bioRxiv preprint doi: https://doi.org/10.1101/2020.07.31.228486; this version posted July 31, 2020. The copyright holder for this preprint (which was not certified by peer review) is the author/funder, who has granted bioRxiv a license to display the preprint in perpetuity. It is made available under aCC-BY 4.0 International license.

Weisblum, Eleftherios Michailidis, Alison W Ashbrook, Eric Waltari, John E Pak, Kathryn E Huey-Tubman, Nicholas Koranda, Pauline R Hoffman, Anthony P West, Jr, Charles M Rice, Theodora Hatziioannou, Pamela J Bjorkman, Paul D Bieniasz, Marina Caskey, and Michel C Nussenzweig. Convergent antibody responses to SARS-CoV-2 in convalescent individuals. Nature, June 2020.

13. Xiangyang Chi, Renhong Yan, Jun Zhang, Guanying Zhang, Yuanyuan Zhang, Meng Hao, Zhe Zhang, Pengfei Fan, Yunzhu Dong, Yilong Yang, Zhengshan Chen, Yingying Guo, Jinlong Zhang, Yaning Li, Xiaohong Song, Yi Chen, Lu Xia, Ling Fu, Lihua Hou, Junjie Xu, Changming Yu, Jianmin Li, Qiang Zhou, and Wei Chen. A neutralizing human antibody binds to the n-terminal domain of the spike protein of SARS-CoV-2. Science, June 2020.

14. Lihong Liu, Pengfei Wang, Manoj S Nair, Jian Yu, Yaoxing Huang, Micah A Rapp, Qian Wang, Yang Luo, Vincent Sahi, Amir Figueroa, Xinzheng V Guo, Gabriele Cerutti, Jude Bimela, Jason Gorman, Tongqing Zhou, Peter D Kwong, Joseph G Sodroski, Michael T Yin, Zizhang Sheng, Lawrence Shapiro, and David D Ho. Potent neutralizing monoclona antibodies directed to multiple epitopes on the SARS-CoV-2 spike. bioRxiv, June 2020.

15. Jesper Pallesen, Nianshuang Wang, Kizzmekia S Corbett, Daniel Wrapp, Robert N Kirchdoerfer, Hannah L Turner, Christopher A Cottrell, Michelle M Becker, Lingshu Wang, We Shi, Wing-Pui Kong, Erica L Andres, Arminja N Kettenbach, Mark R Denison, James D Chappell, Barney S Graham, Andrew B Ward, and Jason S McLellan. Immunogenicity and structures of a rationally designed prefusion MERS-CoV spike antigen. Proc. Natl. Acad. Sci. U. S. A., 114(35):E7348-E7357, August 2017

16. Robert N Kirchdoerfer, Nianshuang Wang, Jesper Pallesen, Daniel Wrapp, Hannah L Turner, Christopher A Cottrell, Kizzmekia S Corbett, Barney S Graham, Jason S McLellan, and Andrew B Ward. Stabilized coronavirus spikes are resistant to conformational changes induced by receptor recognition or proteolysis. Sci. Rep., 8(1):15701, October 2018.

17. Leo Hanke, Laura Vidakovics Perez, Daniel J Sheward, Hrishikesh Das, Tim Schulte, Ainhoa Moliner Morro, Martin Corcoran, Adnane Achour, Gunilla Karlsson Hedestam, B Martin Hällberg, Ben Murrell, and Gerald M McInerney. An alpaca nanobody neutralizes SARSCoV-2 by blocking receptor interaction. June 2020

18. Xaquin Castro Dopico, Leo Hanke, Daniel J Sheward, Murray Christian, Sandra Muschiol, Nastasiya F Grinberg, Monika Adori, Laura Perez Vidakovics, Kim Chang II, Sharesta Khoenkhoen, Pradeepa Pushparaj, Ainhoa Moliner Morro, Marco Mandolesi, Mattias Forsell, Jonathan Coquet, Martin Corcoran, Joanna Rorbach, Soo Aleman, Gordana Bogdanovic, Gerald Mcinerney, Tobias Allander, Chris Wallace, Ben Murrell, Jan Albert, and Gunilla B Karlsson Hedestam. Disease-associated antibody phenotypes and probabilistic seroprevalence estimates during the emergence of SARS-CoV-2. July 2020

19. Viola Schultze, Vicente D'Agosto, Andreas Wack, Deborah Novicki, Juergen Zorn, and Renald Hennig. Safety of MF59 adjuvant. Vaccine, 26(26):3209-3222, June 2008.

20. Barbara Camilloni, Michela Basileo, Stefano Valente, Emilia Nunzi, and Anna Maria lorio. Immunogenicity of intramuscular MF59-adjuvanted and intradermal administered influenza enhanced vaccines in subjects aged over 60: A literature review. Hum. Vaccin. Immunother. 11(3):553-563, 2015.

21. Rebecca J Cox, Gabriel Pedersen, Abdullah S Madhun, Signe Svindland, Marianne Sævik, Lucy Breakwell, Katja Hoschler, Marieke Willemsen, Laura Campitelli, Jane Kristin Nøstbakken, Gerrit Jan Weverling, Jaco Klap, Kenneth C McCullough, Maria Zambon, Ronald Kompier, and Haakon Sjursen. Evaluation of a virosomal H5N1 vaccine formulated with matrix $\mathrm{m}^{\mathrm{TM}}$ adjuvant in a phase I clinical trial. Vaccine, 29(45):8049-8059, October 2011.

22. Qiang Gao, Linlin Bao, Haiyan Mao, Lin Wang, Kangwei Xu, Minnan Yang, Yajing Li, Ling Zhu, Nan Wang, Zhe Lv, Hong Gao, Xiaogin Ge, Biao Kan, Yaling Hu, Jiangning Liu, Fang Cai, Deyu Jiang, Yanhui Yin, Chengfeng Qin, Jing Li, Xuejie Gong, Xiuyu Lou, Wen Shi, Dongdong Wu, Hengming Zhang, Lang Zhu, Wei Deng, Yurong Li, Jinxing Lu, Changgui $\mathrm{Li}$, Xiangxi Wang, Weidong Yin, Yanjun Zhang, and Chuan Qin. Rapid development of an inactivated vaccine candidate for SARS-CoV-2. Science, May 2020

23. Jingyou Yu, Lisa H Tostanoski, Lauren Peter, Noe B Mercado, Katherine McMahan, Shant $H$ Mahrokhian, Joseph P Nkolola, Jinyan Liu, Zhenfeng Li, Abishek Chandrashekar, David R Martinez, Carolin Loos, Caroline Atyeo, Stephanie Fischinger, John S Burke, Matthew D Slein, Yuezhou Chen, Adam Zuiani, Felipe J N Lelis, Meghan Travers, Shaghayegh Habibi, Laurent Pessaint, Alex Van Ry, Kelvin Blade, Renita Brown, Anthony Cook, Brad Finneyfrock, Alan Dodson, Elyse Teow, Jason Velasco, Roland Zahn, Frank Wegmann, Esther A Bondzie, Gabriel Dagotto, Makda S Gebre, Xuan He, Catherine Jacob-Dolan, Marinela Kirilova, Nicole Kordana, Zijin Lin, Lori F Maxfield, Felix Nampanya, Ramya Nityanandam, John D Ventura, Huahua Wan, Yongfei Cai, Bing Chen, Aaron G Schmidt, Duane R Wesemann, Ralph S Baric, Galit Alter, Hanne Andersen, Mark G Lewis, and Dan H Barouch. DNA vaccine protection against SARS-CoV-2 in rhesus macaques. Science, May 2020.

24. Pedro M Folegatti, Katie J Ewer, Parvinder K Aley, Brian Angus, Stephan Becker, Sandra Belij-Rammerstorfer, Duncan Bellamy, Sagida Bibi, Mustapha Bittaye, Elizabeth A Clutterbuck, Christina Dold, Saul N Faust, Adam Finn, Amy L Flaxman, Bassam Hallis, Pau Heath, Daniel Jenkin, Rajeka Lazarus, Rebecca Makinson, Angela M Minassian, Katrina M Pollock, Maheshi Ramasamy, Hannah Robinson, Matthew Snape, Richard Tarrant, Voysey, et al. Safety and immunogenicity of the ChAdOx1 nCoV-19 vaccine against SARS-CoV-2 a preliminary report of a phase $1 / 2$, single-blind, randomised controlled trial. Lancet, July 2020.

25. Lisa A Jackson, Evan J Anderson, Nadine G Rouphael, Paul C Roberts, Mamodikoe Makhene, Rhea N Coler, Michele P McCullough, James D Chappell, Mark R Denison, Laura J Stevens, Andrea J Pruijssers, Adrian McDermott, Britta Flach, Nicole A Doria-Rose, Kizzmekia S Corbett, Kaitlyn M Morabito, Sijy O'Dell, Stephen D Schmidt, Phillip A Swanson, 2nd, Marcelino Padilla, John R Mascola, Kathleen M Neuzil, Hamilton Bennett, Wellington Sun, Etza Peters, Mat Makowski, Jim Albert, Kaitlyn Cross, Wendy Buchanan, Rhonda Pikaart-Tautges, Julie E Ledgerwood, Barney S Graham, John H Beigel, and mRNA-1273 Study Group. An mRNA vaccine against SARS-CoV-2 - preliminary report. N. Engl. J. Med. July 2020.

26. Feng-Cai Zhu, Xu-Hua Guan, Yu-Hua Li, Jian-Ying Huang, Tao Jiang, Li-Hua Hou, Jing Xin Li, Bei-Fang Yang, Ling Wang, Wen-Juan Wang, Shi-Po Wu, Zhao Wang, Xiao-Hong Wu, Jun-Jie Xu, Zhe Zhang, Si-Yue Jia, Bu-Sen Wang, Yi Hu, Jing-Jing Liu, Jun Zhang, Xiao-Ai Qian, Qiong Li, Hong-Xing Pan, Hu-Dachuan Jiang, Peng Deng, Jin-Bo Gou, Xue-
Wen Wang, Xing-Huan Wang, and Wei Chen. Immunogenicity and safety of a recombinant adenovirus type-5-vectored COVID-19 vaccine in healthy adults aged 18 years or older: a randomised, double-blind, placebo-controlled, phase 2 trial. Lancet, July 2020.

27. Philip J M Brouwer, Tom G Caniels, Karlijn van der Straten, Jonne L Snitselaar, Yoann Aldon, Sandhya Bangaru, Jonathan L Torres, Nisreen M A Okba, Mathieu Claireaux, Gius Kerster, Arthur E H Bentlage, Marlies M van Haaren, Denise Guerra, Judith A Burger, Edith E Schermer, Kirsten D Verheul, Niels van der Velde, Alex van der Kooi, Jelle van Schooten, Mariëlle J van Breemen, Tom P L Bijl, Kwinten Sliepen, Aafke Aartse, Ronald Derking, Ilja Bontjer, Neeltje A Kootstra, W Joost Wiersinga, Gestur Vidarsson, Bart L Haagmans, Andrew B Ward, Godelieve J de Bree, Rogier W Sanders, and Marit J van Gils. Potent neutralizing antibodies from COVID-19 patients define multiple targets of vulnerability. Science, June 2020.

28. Emilie Seydoux, Leah J Homad, Anna J MacCamy, K Rachael Parks, Nicholas K Hurlburt, Madeleine F Jennewein, Nicholas R Akins, Andrew B Stuart, Yu-Hsin Wan, Junli Feng, Rachael E Whaley, Suruchi Singh, Michael Boeckh, Kristen W Cohen, M Juliana McElrath, Janet A Englund, Helen Y Chu, Marie Pancera, Andrew T McGuire, and Leonidas Stamatatos. Analysis of a SARS-CoV-2-Infected individual reveals development of potent neutralizing antibodies with limited somatic mutation. Immunity, June 2020.

29. Neeltje van Doremalen, Teresa Lambe, Alexandra Spencer, Sandra Belij-Rammerstorfer, Jyothi N Purushotham, Julia R Port, Victoria Avanzato, Trenton Bushmaker, Amy Flaxman, Marta Ulaszewska, Friederike Feldmann, Elizabeth R Allen, Hannah Sharpe, Jonathan Schulz, Myndi Holbrook, Atsushi Okumura, Kimberly Meade-White, Lizzette Pérez-Pérez, Cameron Bissett, Ciaran Gilbride, Brandi N Williamson, Rebecca Rosenke, Dan Long, Alka Ishwarbhai, Reshma Kailath, Louisa Rose, Susan Morris, Claire Powers, Jamie Lovaglio, Patrick W Hanley, Dana Scott, Greg Saturday, Emmie de Wit, Sarah C Gilbert, and Vincent $\mathrm{J}$ Munster. ChAdOx1 $\mathrm{nCoV}-19$ vaccination prevents SARS-CoV-2 pneumonia in rhesus macaques. bioRxiv, May 2020

30. Kizzmekia S Corbett, Darin Edwards, Sarah R Leist, Olubukola M Abiona, Seyhan BoyogluBarnum, Rebecca A Gillespie, Sunny Himansu, Alexandra Schäfer, Cynthia T Ziwawo, Anthony T DiPiazza, Kenneth H Dinnon, Sayda M Elbashir, Christine A Shaw, Angela Woods, Ethan J Fritch, David R Martinez, Kevin W Bock, Mahnaz Minai, Bianca M Nagata, Geoffrey B Hutchinson, Kapil Bahl, Dario Garcia-Dominguez, Lingzhi Ma, Isabella Renzi, WingPui Kong, Stephen D Schmidt, Lingshu Wang, Yi Zhang, Laura J Stevens, Emily Phung, Lauren A Chang, Rebecca J Loomis, Nedim Emil Altaras, Elisabeth Narayanan, Mihir Metkar, Vlad Presnyak, Catherine Liu, Mark K Louder, Wei Shi, Kwanyee Leung, Eun Sung Yang, Ande West, Kendra L Gully, Nianshuang Wang, Daniel Wrapp, Nicole A Doria-Rose, Guillaume Stewart-Jones, Hamilton Bennett, Martha C Nason, Tracy J Ruckwardt, Jason S McLellan, Mark R Denison, James D Chappell, Ian N Moore, Kaitlyn M Morabito, John R Mascola, Ralph S Baric, Andrea Carfi, and Barney S Graham. SARS-CoV-2 mRNA vaccine development enabled by prototype pathogen preparedness. bioRxiv, June 2020.

31. Stanley A Plotkin. Correlates of protection induced by vaccination. Clin. Vaccine Immunol., 17(7):1055-1065, July 2010

32. Matthias G Pauthner, Joseph P Nkolola, Colin Havenar-Daughton, Ben Murrell, Saman tha M Reiss, Raiza Bastidas, Jérémie Prévost, Rebecca Nedellec, Benjamin von Bredow, Peter Abbink, Christopher A Cottrell, Daniel W Kulp, Talar Tokatlian, Bartek Nogal, Matteo Bianchi, Hui Li, Jeong Hyun Lee, Salvatore T Butera, David T Evans, Lars Hangartner, Andrés Finzi, Ian A Wilson, Richard T Wyatt, Darrell J Irvine, William R Schief, Andrew B Ward, Rogier W Sanders, Shane Crotty, George M Shaw, Dan H Barouch, and Dennis R Burton. Vaccine-Induced protection from homologous tier 2 SHIV challenge in nonhuman primates depends on Serum-Neutralizing antibody titers. Immunity, 50(1):241-252.e6, January 2019.

33. Amarendra Pegu, Bhavesh Borate, Yunda Huang, Matthias G Pauthner, Ann J Hessell, Boris Julg, Nicole A Doria-Rose, Stephen D Schmidt, Lindsay N Carpp, Michelle D Cully, Xuejun Chen, George M Shaw, Dan H Barouch, Nancy L Haigwood, Lawrence Corey, Dennis R Burton, Mario Roederer, Peter B Gilbert, John R Mascola, and Ying Huang. A metaanalysis of passive immunization studies shows that Serum-Neutralizing antibody titer associates with protection against SHIV challenge. Cell Host Microbe, 26(3):336-346.e3, September 2019.

34. Yunlong Cao, Bin Su, Xianghua Guo, Wenjie Sun, Yongqiang Deng, Linlin Bao, Qinyu Zhu, Xu Zhang, Yinghui Zheng, Chenyang Geng, Xiaoran Chai, Runsheng He, Xiaofeng Li, Qi Lv, Hua Zhu, Wei Deng, Yanfeng Xu, Yanjun Wang, Luxin Qiao, Yafang Tan, Liyang Song, Guopeng Wang, Xiaoxia Du, Ning Gao, Jiangning Liu, Junyu Xiao, Xiao-Dong Su, Zongmin Du, Yingmei Feng, Chuan Qin, Chengfeng Qin, Ronghua Jin, and X Sunney Xie. Poten neutralizing antibodies against SARS-CoV-2 identified by High-Throughput Single-Cell sequencing of convalescent patients' B cells. Cell, 182(1):73-84.e16, July 2020.

35. Rui Shi, Chao Shan, Xiaomin Duan, Zhihai Chen, Peipei Liu, Jinwen Song, Tao Song, Xiaoshan Bi, Chao Han, Lianao Wu, Ge Gao, Xue Hu, Yanan Zhang, Zhou Tong, Weijin Huang, William Jun Liu, Guizhen Wu, Bo Zhang, Lan Wang, Jianxun Qi, Hui Feng, FuSheng Wang, Qihui Wang, George Fu Gao, Zhiming Yuan, and Jinghua Yan. A human neutralizing antibody targets the receptor binding site of SARS-CoV-2. Nature, May 2020.

36. Draft landscape of COVID-19 candidate vaccines. https://www.who.int/ publications/m/item/draft-landscape-of-covid-19-candidate-vaccines, Accessed: 2020-7-3.

37. Mark J Mulligan, Kirsten E Lyke, Nicholas Kitchin, Judith Absalon, Alejandra Gurtman, Stephen P Lockhart, Kathleen Neuzil, Vanessa Raabe, Ruth Bailey, Kena A Swanson, and Others. Phase $1 / 2$ study to describe the safety and immunogenicity of a COVID-19 RNA vaccine candidate (BNT162b1) in adults 18 to 55 years of age: interim report. medRxiv, 2020.

38. Jing-Hui Tian, Nita Patel, Robert Haupt, Haixia Zhou, Stuart Weston, Holly Hammond, James Lague, Alyse D Portnoff, James Norton, Mimi Guebre-Xabier, Bin Zhou, Kelsey Jacobson, Sonia Maciejewski, Rafia Khatoon, Malgorzata Wisniewska, Will Moffitt, Stefanie Kluepfel-Stahl, Betty Ekechukwu, James Papin, Sarathi Boddapati, C Jason Wong, Pedro A Piedra, Matthew B Frieman, Michael J Massare, Louis Fries, Karin Lövgren Bengtsson, Linda Stertman, Larry Ellingsworth, Gregory Glenn, and Gale Smith. SARS-CoV-2 spike glycoprotein vaccine candidate NVX-CoV2373 elicits immunogenicity in baboons and protection in mice. June 2020. 
bioRxiv preprint doi: https://doi.org/10.1101/2020.07.31.228486; this version posted July 31, 2020. The copyright holder for this preprint (which was not certified by peer review) is the author/funder, who has granted bioRxiv a license to display the preprint in perpetuity. It is made available under aCC-BY 4.0 International license.

39. Xiaoli Xiong, Kun Qu, Katarzyna A Ciazynska, Myra Hosmillo, Andrew P Carter, Soraya Ebrahimi, Zunlong Ke, Sjors H W Scheres, Laura Bergamaschi, Guinevere L Grice, Ying Zhang, James A Nathan, Stephen Baker, Leo C James, Helen E Baxendale, lan Goodfellow, Rainer Doffinger, John A G Briggs, and The CITIID-NIHR COVID-19 BioResource Collaboration. A thermostable, closed, SARS-CoV-2 spike protein trimer.

40. Dominic Esposito, Jennifer Mehalko, Matthew Drew, Kelly Snead, Vanessa Wall, Troy Taylor, Peter Frank, John-Paul Denson, Min Hong, Gulcin Gulten, Kaitlyn Sadtler, Simon Messing, and William Gillette. Optimizing high-yield production of SARS-CoV-2 soluble spike trimers for serology assays. bioRxiv, May 2020.

41. C L Hsieh, J A Goldsmith, J M Schaub, A M DiVenere, and others. Structure-based design of prefusion-stabilized SARS-CoV-2 spikes. bioRxiv, 2020.

42. Marcella Sarzotti-Kelsoe, Robert T Bailer, Ellen Turk, Chen-Li Lin, Miroslawa Bilska, Kelli M Greene, Hongmei Gao, Christopher A Todd, Daniel A Ozaki, Michael S Seaman, John R Mascola, and David C Montefiori. Optimization and validation of the TZM-bl assay for standardized assessments of neutralizing antibodies against HIV-1. J. Immunol. Methods, 409: 131-146, July 2014. 
bioRxiv preprint doi: https://doi.org/10.1101/2020.07.31.228486; this version posted July 31, 2020. The copyright holder for this preprint (which was not certified by peer review) is the author/funder, who has granted bioRxiv a license to display the preprint in perpetuity. It is made available under aCC-BY 4.0 International license.
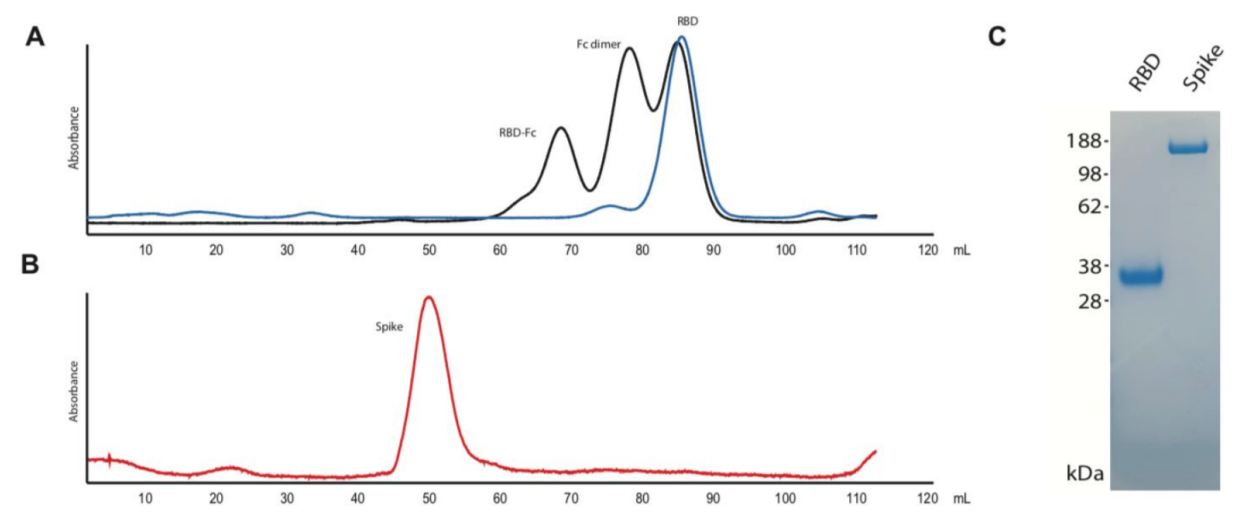

Fig. ED1. SARS-CoV-2 antigen production. A. Size-exclusion chromatograms of affinity purified RBD after enterokinase cleavage digest, before (black) and after (blue) enzyme and Fc removal. B. Chromatogram of affinity purified prefusion-stabilized spike ectodomain. Data from a Superdex S200 16/600 C. SDS-PAGE analysis of purified SARS-CoV-2 RBD and spike immunogens. Maintenance of spike immunogens in the trimeric, prefusion conformation was confirmed by cryo-EM ${ }^{17}$.
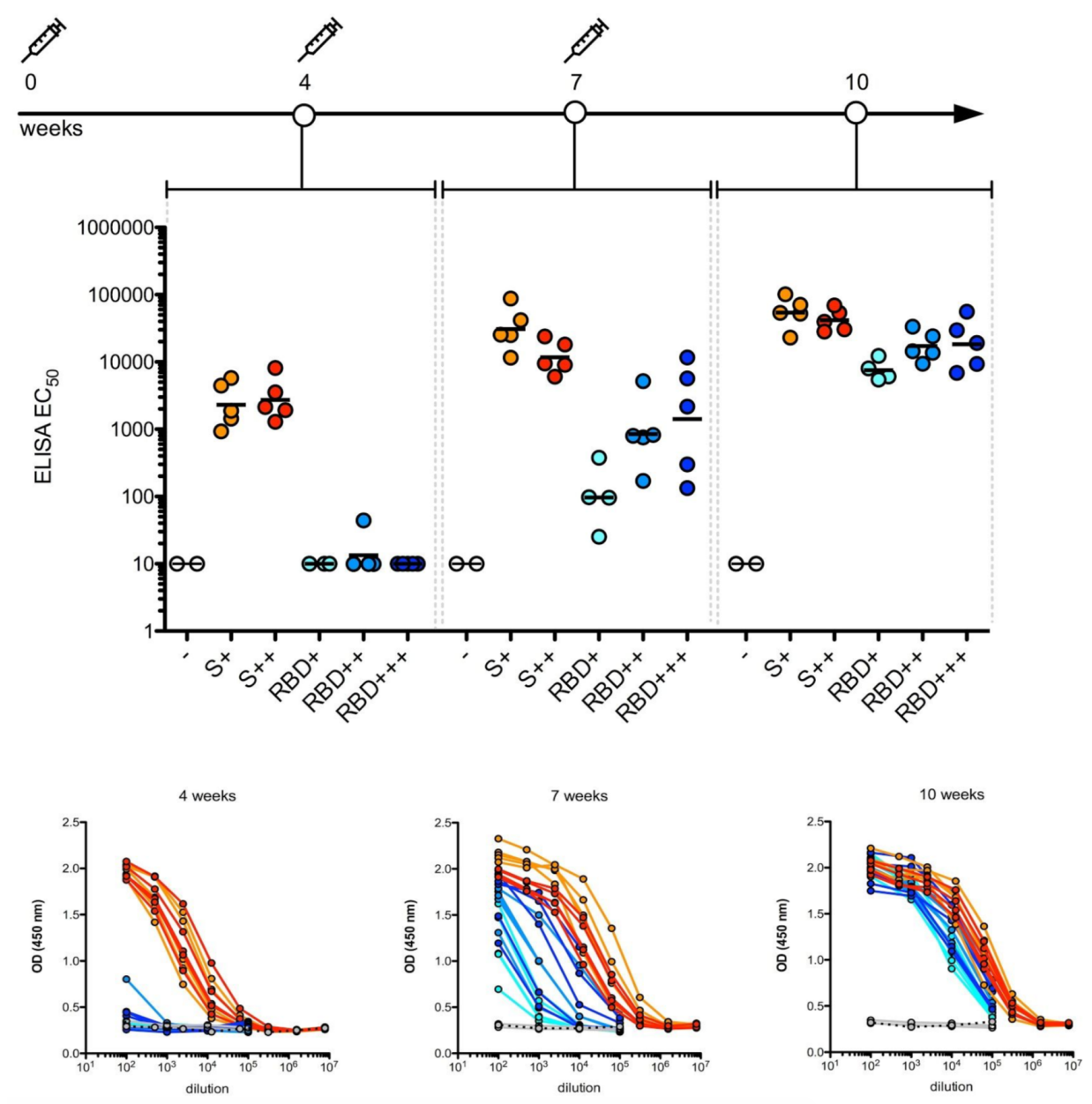

Fig. ED2. Spike and RBD protein subunits are immunogenic in mice. IgG antibodies detected by ELISA in immunized and control mice reveal that stabilized spike and RBD elicit antibody responses after one, two, and three doses. - (unimmunized - open circles); $S+(5 \mu \mathrm{g}$

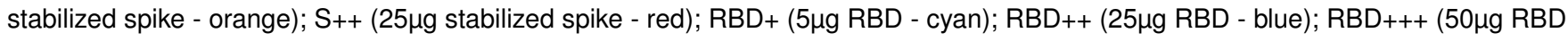
- navy). 
bioRxiv preprint doi: https://doi.org/10.1101/2020.07.31.228486; this version posted July 31, 2020. The copyright holder for this preprint (which was not certified by peer review) is the author/funder, who has granted bioRxiv a license to display the preprint in perpetuity. It is made available under aCC-BY 4.0 International license.

A

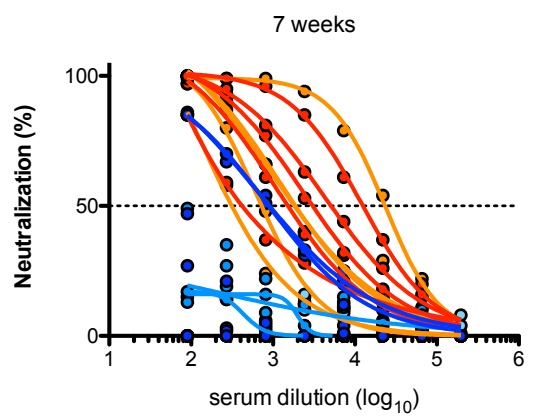

C

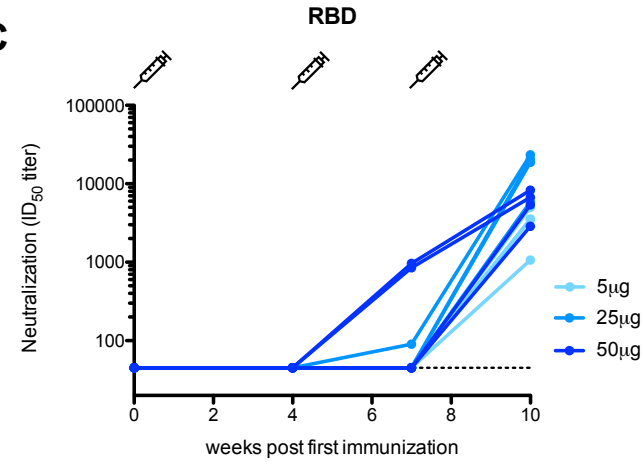

B
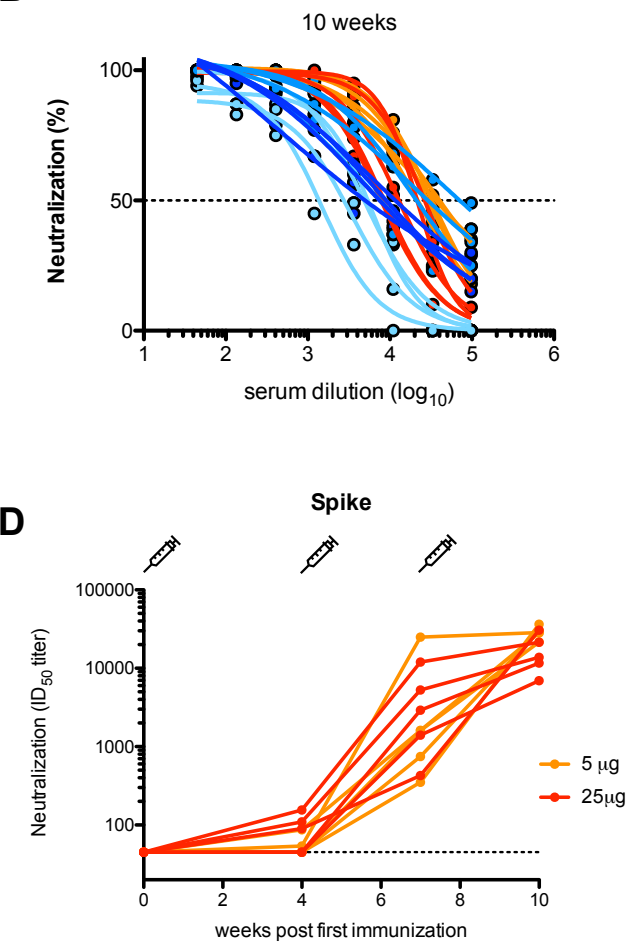

Fig. ED3. A-B. Neutralization curves for mouse sera sampled 7- and 10-weeks post-immunization. Neutralization by serum from mice immunized with RBD are displayed in shades of blue $(5 \mu \mathrm{g}$ doses, cyan; $25 \mu \mathrm{g}$, blue; $50 \mu \mathrm{g}$, navy). Neutralization by serum from

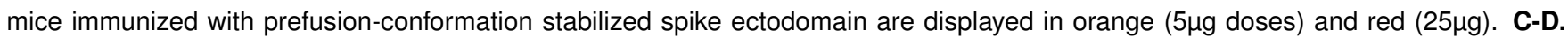
Longitudinal neutralizing antibody ID50 titers in mice immunized with RBD (C) or spike (D). Syringes depict the timing of immunizations (0-, 4- and 7-weeks).

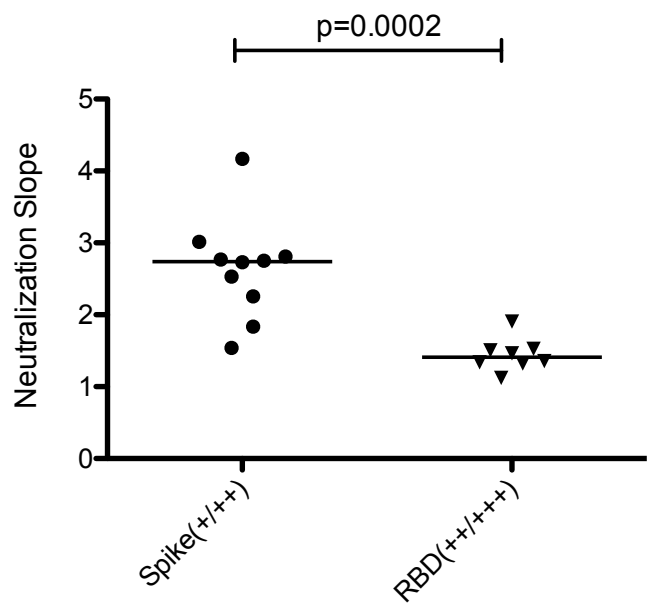

Fig. ED4. Comparison of the slope of neutralizing antibody curves in 10-week serum (see Extended Data Fig 2B) from Spike or RBD (medium and high dose) immunized mice. 
bioRxiv preprint doi: https://doi.org/10.1101/2020.07.31.228486; this version posted July 31, 2020. The copyright holder for this preprint (which was not certified by peer review) is the author/funder, who has granted bioRxiv a license to display the preprint in perpetuity. It is made available under aCC-BY 4.0 International license.

A

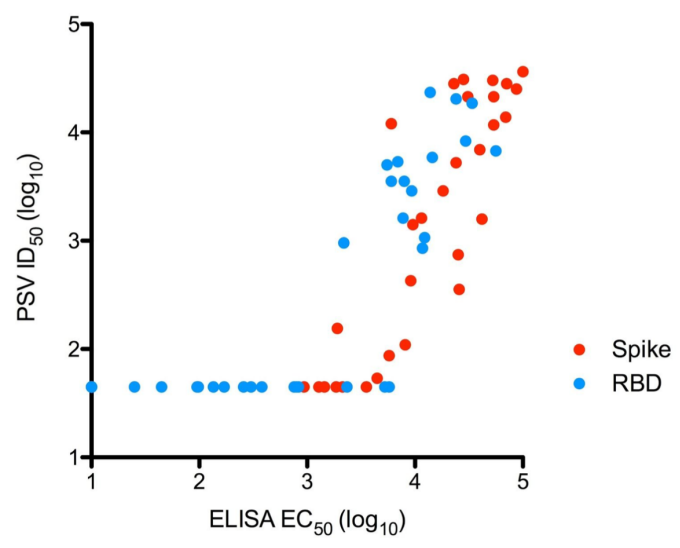

B

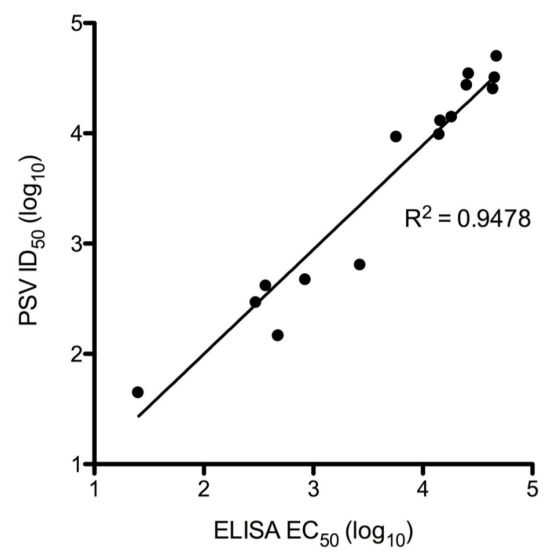

Fig. ED5. Correlation between spike IgG ELISA EC50 values and pseudovirus (PSV) neutralization ID50 titer in immunized mice (A) and macaque (B) sera. Neutralization ID50 below the limit of detection is plotted as the assay's limit of detection (45). 